NOTE: This is an Author's Accepted Manuscript of an article published in CHEMICAL ENGINEERING COMMUNICATIONS [VOL 199, ISSUE 12, (2012)] [copyright Taylor \& Francis], available online at: $\underline{h t t p: / / w w w . t a n d f o n l i n e . c o m / 10.1080 / 00986445.2012 .660712 . ~}$ To cite this article: Santos, R.M. Kawaji, M. (2012). Developments on wetting effects in microfluidic slug flow. Chemical Engineering Communications 199(12), 1626-1641.

\title{
Developments on Wetting Effects in Microfluidic Slug Flow
}

\section{R. M. SANTOS ${ }^{1}$, M. KAWAJI ${ }^{2}$}

${ }^{1}$ Department of Chemical Engineering, Katholieke Universiteit Leuven, Willem de

Croylaan 46, Leuven, 3001, Belgium. Email: rafael.santos@alumni.utoronto.ca

${ }^{2}$ Department of Mechanical Engineering, The City College of New York, 160

Convent Avenue, New York, NY 10031, USA. Email: kawaji@me.ccny.cuny.edu

Wetting effects form a dimension of fluid dynamics that becomes predominant, precisely controllable and possibly useful at the micro-scale. Microfluidic multiphase flow patterns, including size, shape and velocity of fluidic particles, and mass and heat transfer rates are affected by wetting properties of microchannel walls and surface tensions forces between fluid phases. The novelty of this field, coupled to difficulties in experimental design and measurements, means that literature results are scarce and scientific understanding is incomplete. Numerical methods developed recently have enabled a shortcut in obtaining results that can be perceived realistic, and that offer insight otherwise not possible. In this work the effect of the contact angle on gas-liquid two-phase flow slug formation in a microchannel Tjunction was studied by numerical simulation. The contact angle, varied from 0 to 140 degrees, influenced the interaction of the gas and liquid phases with the channel wall, affecting the shape, size and velocity of the slugs. The visualisation of the cross-sectional area of gas slugs allowed for insight 
into the existence of liquid flow along rectangular microchannel corners, which was affected by the contact angle and determined the occurrence of velocity slip. The velocity profile within the gas slugs was also found to change as a function of contact angle, with hydrophilic channels inducing greater internal circulation, compared to greater channel wall contact in the case of hydrophobic channels. These effects play a role in heat and mass transfer from channels walls and highlight the value of numeral simulation in microfluidic design.

Keywords Microchannel; Contact angle; Wetting; Taylor slug; Computational fluid dynamics; T-junction

\section{Introduction}

The formation of Taylor slugs in microchannels can be applied in a variety of Lab-on-a-chip and other microfluidic devices, making technological innovations possible that could not otherwise be accomplished in conventional scales. There are some specific advantages to performing certain types of analytical and chemical work at the microfluidic scale. Microchannel reactors, for example, offer the advantage of having extremely high surface to volume ratio, which results in increased heat and mass transfer rates. Taylor slugs have also been shown to increase transverse mass and heat transfer because of recirculation within the liquid plugs and the reduction of axial mixing between liquid plugs (Irandoust and Andersson, 1989).

At the micro-scale, surface tension forces (Equation 1) become more important, being able to balance and even overcome gravitational, inertial, shearing and drag forces. This allows the control of fluidic trajectories and interactions not only by designing channel geometries and controlling flow regimes, but also by the tuning of wetting properties, which affect the formation and behaviour of interfacial boundaries between fluids and contact lines with the channel walls. For instance, the occurrence of a continuous phase film along channels walls is desirable to increase fluid-fluid contact area, or to increase wall-fluid contact of one fluid while limiting that of the other fluid. Likewise the absence of such film or the formation of droplets by prevention of fluid spreading can be interesting in some applications. Feng et al. (2009) used successive flow-focussing microfluidic cross-sections of different wetting characteristics to first form a typical two-phase slug flow (an emulsion), and later break the emulsion into droplet pairs surrounded by a third liquid 
continuous phase. The different wetting properties of the channels ensured that in each channel region only one fluid phase preferentially wetted the wall. Takei et al. (2007) developed multi-step Laplace valves based on photocatalytic patterning and tuning of the surface wettability of microchannels. The working principle relies on the pressure drop caused by capillary forces imposed on the meniscus at the front end of a liquid plug, and is a function of the local contact angle.

This paper presents numerical simulation results on the effect of the contact angle on slug formation in a microchannel T-junction using ANSYS FLUENT's VOF model, as an extension of the study previously reported by the present authors in Santos and Kawaji (2010). The obtained results are meant to provide direction in future research. Results and observation are compared to other recent findings with the aims of bringing consensus into the field, providing insight into the microfluidic flow physics not yet fully understood, and assessing the capabilities and deficiencies of current CFD modelling techniques for microfluidic multiphase flow.

\section{Background}

\section{Fundamental Wetting Principles}

Young (1805) was the original researcher to derive an expression for the static contact angle $(\theta)$, by assuming that each of three material boundaries (solid/liquid, solid/gas and gas/liquid) has a particular constant surface tension $(\sigma)$. Young reasoned that in order for the system to be at equilibrium, the horizontal components of the tensions must add up to zero at the contact line (the point where the three materials meet). Young's equation relates the contact angle and interfacial tensions according to Figure 1 and takes the following form:

$$
\sigma_{G L} \cdot \cos (\theta)=\sigma_{S G}-\sigma_{S L}
$$

It is usual to term surfaces that form contact angles in the range $0 \leq \theta<90^{\circ}$ as wetting (or hydrophilic), and those for which the contact angle lies in the range $90^{\circ} \leq \theta \leq 180^{\circ}$ are called non-wetting (or hydrophobic). The contact angle described by Young (1805) is referred to as the static contact angle. However, in experiments where the contact line is moving, it is possible to observe the existence of two different contact angles, one on the advancing side $(U>0)$ and another on the receding side $(U<0)$, where $U$ is the axial fluid 
velocity. The value of $\theta$ in the limit of $U \rightarrow 0$ when approaching from the $U>0$ side is denoted as an advancing contact angle $\left(\theta_{A}\right)$, and when approaching from the $U<0$ side it is called a receding contact angle $\left(\theta_{R}\right)$. In certain systems there exists an interval $\left\{\theta_{R}, \theta_{A}\right\}$ within which the contact line does not move, and this non-uniqueness in contact angle value is referred to as contact angle hysteresis (Dussan, 1979). Moreover for non-ideal surfaces, possessing, for example, roughness or residual impurities, pinning of the contact line, leading to increased resistance to movement and stick-slip behaviour, has been observed (Schäffer and Wong, 2000).

While having a significant implication, the existence of the dynamic contact angle is not always easy to take into account in experiments and in computational modeling. This is because the only contact angle that can be known a priori is the static contact angle. In a microfluidic system, it could be theoretically possible to measure the contact angles of moving slugs and obtain some type of correlation of contact angle as a function of fluid and/or slug velocity. However the difficulties in contact angle measurement at the micro-scale and the complexity of the multiphase flow fields in microchannels, including time-dependency, variability and inconsistencies (Schäffer and Wong, 2000; Kawahara et al., 2002), make this type of procedure less than favourable.

Skartsis et al. (1992) performed experiments in the Capillary number $\left(\mathrm{Ca}=\mu_{c} U / \sigma\right)$ range of $10^{-6}$ to $10^{-4}$, and concluded that in this range the static contact angle provides an excellent approximation to the dynamic value. In the present study, numerical simulation was performed for $\mathrm{Ca}$ in the order of $10^{-3}$; therefore there may be a marginal difference in the contact angle value. For simplification and software limitations, however, the static value was considered sufficient for numerical simulation in the present work.

The value of the static contact angle formed by two fluids on a solid surface is nearly always not dependent solely on the type of material that makes up the surface. Several factors can change the behaviour of the contact line, or at least change the value of the apparent (visually observed) contact angle. In fact, the chemical character of the first monolayer of molecules on a surface can have a significant impact on the contact angle. Dussan (1979) found that by submerging a smooth, clean solid surface in a bath comprised of a polar solute dissolved in a nonpolar solvent (heptadecylamine in mineral oil) the nature of the surface becomes masked to such an extent that the adsorbed molecules dominate the determination of the contact 
angle. In such instance, even if different solid materials were used, the measured contact angle would not change.

The surface roughness is another variable that impacts the measured value of the contact angle. While at the microscopic scale the contact line behaviour does not change, surface imperfections shift the contact line upwards or downwards, resulting in a measured contact angle that appears different from the expected value for a smooth identical material. Hence the value measured in experiments is not necessarily the true contact angle as described by Equation (1). The two contact angles can be simply related to each other by a roughness ratio ( $\Gamma=$ true wetted surface area / apparent surface area) according to Equation (2) (Moita and Moreira, 2003):

$$
\cos \left(\theta_{\text {apparent }}\right)=\Gamma \cdot \cos \left(\theta_{\text {true }}\right)
$$

A useful discussion of the effect of surface roughness on the contact angle, as well as a thermodynamic approach to describing the problem, is made by Wenzel (1936). It is theorized that wetting replaces an area of solid-gas interface by an equal solid-liquid area accompanied by a liquid-gas interface, resulting in a net decrease or increase in total surface energy. If the wetted area has a lower specific energy (hydrophilic), a drop of liquid will spontaneously spread on it, releasing energy that is consumed by the expansion of the liquid-gas surface until equilibrium is reached and the static contact angle is formed. For a rough surface of the same material, the amount of wetted surface for the same wetted area is larger; consequently a greater net decrease in energy results, inducing more rapid and extensive wetting. The same analysis is valid for the case of a hydrophobic surface, although in that case the rough surface will wet less.

The main reason that the contact angle is important in two-phase flow problems, especially those involving slug flow in microfluidic channels, is that it not only affects to the shape of Taylor slugs, but it also affects the ease with which they travel in the channel (i.e. the slug velocity). To understand this mechanism it is useful to examine a simple case, described by West (1911). Suppose there is a meniscus within a capillary tube formed by air and a liquid phase. The pressure drop $(\Delta P)$ that must be imposed to produce a volumetric flow rate $Q$ over a distance $L$ in a channel of radius $r$ is given by Equation (3):

$$
\Delta P=\frac{8 Q \mu L}{\pi r^{4}}-\left(\frac{2 \sigma}{r}\right) \cdot \cos (\theta)
$$


This means that for a constant pressure system the flow rate in a microchannel is affected by the value of the contact angle $(\theta)$ and the interfacial tension $(\sigma)$; conversely for a constant flow system the pressure drop would be affected. This is the same principle used in designing Laplace valves like those in Takei et al. (2007).

\section{Wetting in Experimental Microfluidics}

Ide et al. (2008) conducted experimental work on the effect of wetting properties of a microchannel on the occurrence of slug flow and the characteristics of the slug flow (liquid plug/gas slug lengths and velocities, void fraction, and pressure drop). They used a T-junction to mix the gas/liquid streams connected to a straight microchannel, both of $100 \mu \mathrm{m}$ circular diameters. The fused silica microchannel was silanized with trimethylsilane groups to render it non-wetting, and was compared to the original untreated microchannel. They observed gas slugs with flat ends in the non-wetting case, compared to gas slugs with typical semi-hemispherical ends in the wetting case. At higher superficial flow velocities the slug flow pattern changed to ring flow, but the transition for non-wetting channels occurred at higher liquid superficial velocities $(>0.2 \mathrm{~m} / \mathrm{s})$. Also, the observed liquid ring for the non-wetting case was thinner. The use of nonwetting channels also resulted in higher void fraction, slower gas slugs, shorter liquid plugs and higher pressure drop.

Barajas and Panton (1993) and Kandlikar et al. (2010) have reported on shifts in flow pattern regimes due to changes in wetting properties of capillaries and microchannels, respectively. Barajas and Panton (1993) found that for partially wetting channels $\left(30^{\circ}<\theta<90^{\circ}\right)$, a new flow regime called rivulet flow appeared, replacing wavy flow. More significantly, for non-wetting channels $\left(\theta=106^{\circ}\right.$, made of FEP fluoropolymer) several flow pattern transition boundaries shifted, particularly from plug and slug flows to bubble, annular and wavy/rivulet flows. Kandlikar et al. (2010) report similar trends, but for microchannel flow. They noticed different regimes for three classes of wetting properties: hydrophilic $\left(15^{\circ}\right)$, partially wetting $\left(60^{\circ}\right)$ and hydrophobic $\left(100^{\circ}\right.$, silanized Lexan). While in hydrophilic channels the flow transition due to increasing gas superficial velocities was from slug to film flow, in partially wetting channels a new flow pattern termed mist (similar to rivulet) appeared for high gas velocities. More interestingly, for hydrophobic channels the 
predominant flow pattern became droplet flow, combined with slug and film flows at low and high gas velocities, respectively. Ody (2010) observed at low capillary numbers (approximately $5 \times 10^{-4}$ ) the effect of wetting properties on the shape of the menisci at the nose and tail of gas slugs and liquid plugs. For instance, the curvature at the tail of gas slugs changed from convex out to concave in, in reference to its body center.

Fang et al. (2010) studied the effect of microchannel wall hydrophobicity on the flow patterns formed by condensation of steam, and on the heat transfer rates. Their experiments were conducted in silicon channels of 500 × $200 \mu \mathrm{m}$ size, treated by Molecular Vapor Deposition (MVD) to give three surface types: hydrophilic $\left(25^{\circ}\right)$, semi-hydrophobic $\left(91^{\circ}\right)$ and hydrophobic $\left(123^{\circ}\right)$. Hydrophobicity significantly affected the condensation flow pattern, both in the upstream condensation region (film/stratified flow and dropwise flow were dominant in the hydrophilic and hydrophobic channels, respectively), and the downstream two-phase flow region (bubbly flow and slug flow were dominant in the hydrophilic and hydrophobic channels, respectively). Heat transfer rates were found to be lower in the hydrophilic channel due to filmwise condensation. In this case the liquid film thickness, which had poor thermal conductivity, increased the thermal resistance. The pressure drop in the hydrophobic channel, however, was found to be higher due to the greater pressure gradient needed to overcome the resistance of slug motion due to contact angle hysteresis.

\section{Wetting in Computational Microfluidics}

Recent advances in the field of numerical analysis, and more specifically multiphase computational fluid dynamics (CFD), allow for information about local parameters such as velocity profiles, volumetric mass fractions and interface configurations to be easily obtained, whereas in experimental work such data are difficult or not possible to be measured. Several numerical studies using different CFD models have been performed, including the Volume of Fluid (VOF), Level Set, and Lattice Boltzman methods. Previous work by this group (Santos and Kawaji, 2010) correlated the 3-D numerical and experimental results of air-water slug formation in a microfluidic T-junction. It was shown that 3-D modeling is critical to capture the correct surface tension effects on slug break-up and the Laplace pressure effect on slug shape.

Several works using full three-dimensional or two-dimensional axisymmetrical computation have come out in recent years on the subject of wetting effects in microchannel Taylor slug, bubbly and droplet flow. 
Rosengarten et al. (2006) studied the effect of wetting properties of the fluids relative to the channel walls on two-phase flow patterns in microchannel contractions. This geometry was chosen to promote interface contact with the channel wall when a droplet entered the microfluidic contraction. They explained that a drop that is initially detached from a wall, surrounded by a lubricating film, must drain the continuous fluid film away before it can interact with the wall, and that the corner of the contraction minimized the required fluid drainage. Numerical modeling showed significant effect of contact angle on slug deformation in the case of high viscosity ratio $\left(\lambda=\mu_{d} / \mu_{c}\right)$.

Gupta et al. (2009) focussed on the hydrodynamics of slug flow in a straight channel. They postulated that Taylor bubbles are not affected by the contact angle due to the presence of a thin liquid film, except in cases where the two phases mix from separate streams (i.e. slug formation effect) or for non-circular geometries. Such cases describe, however, the vast majority of experimental microfluidic work on slug flow, given micromachining of perfectly circular channels is atypical (especially with lithography and etching techniques) and mixing of streams from T-,Y- and cross-junctions is common. Chung et al. (2004) compared gas-liquid two-phase flows in circular and square microchannels. They pointed out the existence of liquid at the corners of square microchannels, which prevents the formation of the ring film structure reported by Kawahara et al. (2002) and Serizawa et al. (2002).

Raj et al. (2010) and Yong et al. (2011) modelled liquid-liquid slug flow formation in rectangular microchannel junctions $(50-100 \times 33 \mu \mathrm{m}$, and $300 \times 600 \mu \mathrm{m}$, respectively). The first study used silicone oil and water; the latter opted for kerosene and water. Likewise different CFD codes were used: Fluent's 3D VOF, and in-house developed Lattice Boltzman, respectively. Raj et al. (2010) modelled the formation of slugs and compared their numerical results to experimental results in literature. The contact angle value was found to be a critical parameter in matching the data. By varying the contact angle from 0 to $120^{\circ}$, the flow pattern that best fitted the experimental observations was found. Yong et al. (2011) similarly found the contact angle to change the microfluidic flow pattern: (i) slug flow occurred for values between 20 and $40^{\circ}$; (ii) the ends of the slugs became straightened at $90^{\circ}$; and (iii) stratified flow was produced for values in the range of 150 to $160^{\circ}$. Kumar et al. (2007) found that the contact angle value (varied from 25 to $150^{\circ}$ ) affected the length of gas-liquid slugs formed in a curved circular microchannel, with the shortest slugs forming at $90^{\circ}$. 
Özkan et al. (2010) modelled the formation of a gas slug from a small orifice growing into a liquid filled rectangular microchannel. The fluids considered were nitrobenzene and hydrogen gas, and the microchannel surfaces considered were carbon and stainless steel. The contact angle values of these two materials were measured experimentally to be $5^{\circ}$ and $12^{\circ}$, respectively. This system was chosen in view of modelling the multiphase catalytic hydrogenation of nitrobenzene to aniline. The microchannel dimensions were $400 \mu \mathrm{m} x$ $1 \mathrm{~mm}$, and the computational mesh size used was $20 \mu \mathrm{m}$. A finer mesh was recommended to better capture the liquid film, which was observed along the narrower channel dimension but not along the wider dimension; implementation of a finer mesh was avoided due to the high computational cost. While the contact angle was found to have little effect on the fluid dynamics when varied below $15^{\circ}$, above $90^{\circ}$ the gas phase was seen to spread on the channel wall. Such behaviour was considered to be undesirable for the chemical reaction since higher mass and heat transfer rates were thought to be achieved in the presence of a thin liquid film. This study also highlighted the need for implementation of dynamic contact angle and slip boundary conditions for more accurate simulation of wetting effects in microchannels.

\section{Numerical Methods}

FLUENT 6.2 CFD software was used to simulate Taylor slug formation in a microfluidic T-junction. The Volume of Fluid (VOF) model (Hirt and Nichols, 1981) in three-dimensional form was used, which enabled capturing and tracking the precise location of the interface between the fluids. Governing equations are reported in literature (Santos and Kawaji, 2010; Raj et al., 2010, Özkan et al. 2010). A segregated axisymmetric time-dependent unsteady solver was used along with the implicit body force formulation. For discretization the PRESTO! (Pressure staggering options) scheme was used for pressure interpolation, the PISO (Pressure-implicit with splitting of operators) scheme was used for pressure-velocity coupling, and the second-order up-wind differencing scheme was used for the momentum equation. Air was designated as the primary phase and water as the secondary phase. Wall adhesion was enabled so that the contact angle could be prescribed and a constant surface tension value ( $\sigma=73.5 \mathrm{dyn} / \mathrm{cm}$ for air-water) was inputted.

The microchannel geometry consistent of a T-junction with inlet channel widths of $111 \mu \mathrm{m}$ for liquid stream (main line) and $118 \mu \mathrm{m}$ for gas stream (branch), and outlet channel width of $108 \mu \mathrm{m}$. The channel 
depth (z-direction) was $119 \mu \mathrm{m}$ (axisymmetric $=2$ x $59.5 \mu \mathrm{m})$ for all channels. These dimensions, along with the slightly irregular shape of the T-junction, were chosen to replicate the microchannel geometry previously used experimentally by this group (more detail on the channel geometry can found in Santos and Kawaji (2011)). The computational mesh was prepared using the Gambit 2.3 software (Fluent Inc.). Quadrilateral elements were used with the paved meshing scheme (which generates an unstructured mesh that best contoured the T-junction geometry) and a mesh spacing of $5.67 \mu \mathrm{m}$. The cross-sectional mesh at the symmetry plane $(z=56.5 \mu \mathrm{m})$ of the T-junction, which consisted of 4,945 facets, is shown in Figure 2 . The full three-dimensional computational domain for the microfluidic channel consisted of 98,000 computational cells. Grid and time-step independence studies are detailed in the accompanying Supplementary Materials.

Gupta et al. (2009) proposed that Taylor bubbles are always surrounded by a thin liquid film and that a fine enough mesh is needed to capture the film. They reviewed numerous numerical studies and pointed to the lack of mesh refinement near the wall in the majority of studies. Their own numerical work showed a significant effect on the slug-wall interaction and hydrodynamics by the use of refined near-wall mesh. However, they also state that in some particular cases, such as transient mixing of gas and liquid from separate streams or flow in non-circular geometries, the dry-out scenario (reported by Serizawa et al. (2002)) is possible. In their work the computational geometry consisted of a straight channel with gas and liquid phases entering the channel in a gas-core liquid-ring fashion. They then tracked the movement of gas slugs to conclude the slug does not interact with the wall and that contact angle is not a crucial factor in microfluidic two-phase flow. The present work deals with mixing of gas and liquid from separate streams, whereby both fluids touch the wall at the T-junction, and also with non-circular channels. As such, given the appropriate wettability conditions, it is expected that non-existence of a liquid film is possible under certain scenarios in this work. Moreover, the mesh size used here (in computationally expensive 3D as opposed to 2Daxisymmetrical) is similar to the $5 \mu \mathrm{m}$ mesh size used in Gupta et al. (2009), and significantly less than the $20 \mu \mathrm{m}$ size used by Özkan et al. (2010). Also the channel diameter in the present study is truly microfluidic in the order of $100 \mu \mathrm{m}$ (as in Serizawa et al. (2002)), which is much less than the $500 \mu \mathrm{m}$ channel used by Gupta et al. (2009), and hence local effects like the Laplace pressure are accentuated. The generalization that Taylor bubbles are always surrounded by a liquid film, and that any numerical study that does not show this is 
inaccurate, must be taken with caution. More crucial is the use of 3-D modeling, which some authors overlook, as it has a significant impact on hydrodynamics (Santos and Kawaji, 2010).

The velocity of gas slugs $\left(v_{\mathrm{S}}\right)$ was calculated using a user-defined function (UDF) routine (Santos and Kawaji, 2011) to average the axial velocity (x-direction) of the gas phase contained within the gas slug according to Equation (4), and the volume of gas slugs $\left(V_{S}\right)$ was calculated by the summation of the gas phase volume fraction $\left(\Omega_{G}\right)$ contained in the range of computation (cellrange) according to Equation (5):

$$
\begin{gathered}
v_{S}=\frac{\sum_{i=1}^{\text {cellrange }} \Omega_{G, i} \cdot V_{\text {cell }, i} \cdot v_{x, i}}{\sum_{i=1}^{\text {cellrange }} \Omega_{G, i} \cdot V_{\text {cell }, i}} \\
V_{S}=\sum_{i=1}^{\text {cellrange }} \Omega_{G, i} \cdot V_{\text {cell }, i}
\end{gathered}
$$

\section{Results and Discussion}

The contact angle $(\theta)$ is an important parameter in the slug formation process, as it determines the characteristics of the interaction of the gas and liquid slugs with the channel wall. In the FLUENT code, the user specifies the value of the static contact angle and it is used to solve the wall-adhesion equations, which determine the shape of the fluid interface at and near the wall. In the present study it was of interest to investigate the effect of using different contact angle values on the gas-liquid slug formation in a microchannel T-junction. In particular, it was desirable to know if the flow pattern (slug size and shape) or flow parameters (velocity profile and mean slug velocity) would change significantly as a function of the contact angle.

To study the effect of the contact angle on slug formation, the static contact angle was varied from $0^{\circ}$ to $140^{\circ}$. The inlet superficial velocities of liquid and gas were kept constant for all runs at respectively $U_{L}=$ $0.168 \mathrm{~m} / \mathrm{s}$ and $U_{G}=0.237 \mathrm{~m} / \mathrm{s}$, median values of experiments conducted in Santos and Kawaji (2010). The corresponding outlet superficial velocity $\left(U_{\text {out }}\right)$, equal to the volumetric sum of inlet flow rates divided by the outlet channel cross-sectional area, in this case is $0.432 \mathrm{~m} / \mathrm{s}$. The corresponding Capillary number is $5.9 \cdot 10^{-3}$. 
Images of the flow patterns produced with nine values of contact angle are displayed in Figure 3, and the corresponding numerical data is presented in Table 1. In the accompanying Supplementary Materials, videos of slug formation for three contact angle values $\left(31^{\circ}, 90^{\circ}\right.$ and $\left.120^{\circ}\right)$ are provided. From Figure 3 it is seen that the radius of curvature at the end of the gas slug increases as the contact angle increases. At a value of $\theta=90^{\circ}$, the interface becomes completely vertical, at a right-angle with the channel wall. It can be observed that the gas slug shape changes from convex out to concave in as the contact angle increases further.

For most cases in Figure 3 the apparent contact angle of the advancing gas slug end appears smaller than that of the receding end, indicating a certain degree of contact angle hysteresis prediction, despite the static contact angle value used. The shape of a gas slug can, besides being related to contact angle, be related to the difference between the static pressure inside a gas slug to that in the surrounding liquid phase. The YoungLaplace equation for a meniscus (Equation 6) predicts the change in interface curvature as a function of the static pressure difference (the Laplace pressure), and can be applied to describe the shape of, and the pressure balance at the ends of Taylor slugs in a microchannel. A large pressure difference reduces the radius of curvature $\left(R_{C}\right)$, and hence produces a more curved interface. As the pressure difference is lowered, the interface flattens.

$$
P_{\text {inside }}-P_{\text {outside }}=\frac{2 \sigma \cos \theta}{R_{C}}
$$

Close-up images of fast and slow gas slugs produced numerically with the present model are shown in Figure 4 in the form of static pressure contours. In this case the contact angle value used was $36^{\circ}$ (equal to the experimental value in Santos and Kawaji (2010)). It can be seen that the interface curvature is more accentuated at the front nose of the gas slug with slug velocity $\left(v_{S}\right)$ equal to $1.379 \mathrm{~m} / \mathrm{s}$, where the difference in static pressure is large (in the order of $3.3 \mathrm{kPa}$ ). At the rear end of the same gas slug the pressure difference is smaller (approximately $2 \mathrm{kPa}$ ) and the interface shape is attenuated, becoming more hemi-spherical. For the slower gas slug with $v_{S}=0.086 \mathrm{~m} / \mathrm{s}$ the pressure difference at either end of the gas slug is approximately the same $(2 \mathrm{kPa})$, and hence the shapes of the interfaces are identically hemi-spherical. For comparison, an air/water meniscus with radius of curvature equal to the channel hydraulic radius would produce a pressure difference of $2.2 \mathrm{kPa}$. These numerical results confirm that hydrodynamics can significantly affect the slug 
shape, and that the static contact angle used in the model does not necessarily force the interface curvature to remain rigid.

Furthermore, at the T-junction in Figure 3, it is possible to see that for the hydrophilic cases, the next slug that begins to form emerges more towards the center of the liquid flow. For the hydrophobic contact angles, $\theta \geq 90^{\circ}$, the gas stream attaches to the top wall of the outlet channel and moves along it as the gas slug expands. At $\theta=140^{\circ}$, the gas slug has not detached from the gas inlet by the time it reaches the end of the computational domain. It would appear possible at such hydrophobic channel conditions that the flow pattern could change to dropwise flow rather than slug flow, as experimentally observed by Kandlikar et al. (2010) and Fang et al. (2010).

The cross-sections of gas slugs along the geometrically centered y-z plane for $\theta=0^{\circ}, 20^{\circ}$ and $40-120^{\circ}$ are shown in Figure 5. Two sets of images are included. The top row contours are produced using a gas volume fraction $\left(\Omega_{G}\right)$ threshold of 0.5 , much in the way a Level Set model would be represented. While producing a smooth slug contour, the disadvantage in this case is the absence of visualizing the thin liquid film along the walls. For this reason the bottom row in Figure 5 is plotted, showing the values of $\Omega_{G}$ at each computational facet without node interpolation. When the value of $\Omega_{G}$ is $<1$, an amount of liquid phase is present in the facet, evidently located closest to the channel wall or the neighbouring facet containing solely liquid phase.

The difference between the slugs formed at $0^{\circ}$ and $20^{\circ}$ is mainly the film thickness around the gas slug, which can be inferred from the gas-phase cross-sectional area coverage $\left(A_{G}\right)$ calculated based on Equation (7):

$$
A_{G}=\frac{\sum_{i=1}^{380} \Omega_{G, i}}{380}
$$

$A_{G}$ is greater at $\theta=20^{\circ}(89 \%)$ than at $\theta=0^{\circ}(85 \%)$. An exact film thickness is difficult to express, as the liquid film is thicker at the channel corners and thinner on the side walls due to the rectangular channel crosssectional shape. It is seen that for $\theta>40^{\circ}$ the gas phase completely fills the channel cross-section. As a result, the gas slugs in hydrophobic channels cannot travel faster than the liquid phase, and therefore move at the same velocity $\left(v_{S}\right)$ as the total outlet superficial velocity $\left(U_{\text {out }}\right)$, as shown in Table 1 . Where the contact angle is lower, the gas slugs are able to travel faster than the liquid phase (i.e. velocity slip occurs), and hence their 
velocities are greater than $U_{\text {out }}$. These results agree with experimental observations of Ide et al. (2008), who also concluded non-wetting channels result in slower gas slugs and higher void fraction (a product of the elimination of velocity slip). Velocity slip was found to be a probable cause of the discrepancy between numerical and experimental results by Santos and Kawaji (2010), where experimental data suggested significantly higher velocity slip. Therefore the use of hydrophobic channels may provide a more accurate means of designing two-phase flow microchannels by numerical simulation. Moreover, hydrophobic channels also provide the opportunity to enhance the contact area of the gas slug with the channel wall, which can be of interest for the development of microfluidic systems where direct heat or mass exchange with the channel wall or a reaction at the channel wall occurs.

Table 1 also provides the volumes $\left(V_{S}\right)$ of the gas slugs formed. It can be seen that the gas slug formed in the case of $\theta=20^{\circ}$ is smallest, while at $\theta=40^{\circ}$ it is largest. Increased interface instability and stability, respectively, likely play a role in this difference, causing earlier or later slug break-up. The shape of the Tjunction corners, here not perfectly square to represent the experimental channel in Santos and Kawaji (2010), also may play a role in determining slug break-up timing. The slug volume then decreases as $\theta$ increases beyond $40^{\circ}$. It can be concluded that the contact angle affects the size of gas slugs. Like Kumar et al. (2007), the minimum value of slug length $\left(L_{s}\right)$ occurs at $\theta=90^{\circ}$, due to the straight rather than curved interface of the slug nose and tail. It is evident that due to interface curvature changes the slug length is not the most appropriate indicator of slug size; thus the volume-based approach reported here is recommended for future studies.

The contact angle is also found to affect the velocity field, especially the recirculation within the gas slug. Figure 6 compares the cases using $0^{\circ}$ and $90^{\circ}$, where the outlet superficial velocity $\left(U_{\text {out }}\right)$ was $0.432 \mathrm{~m} / \mathrm{s}$. These contour plots are made from the outlet flow channel, where the gas slug fills the majority of the visible field. Also, the plots are made at the diagonal plane across the channel, i.e. from one lower channel corner to the opposite upper channel corner. It can be seen from the comparison between Figures 6(a) and 6(b) that the recirculation in (a) is more intense, given the higher axial velocity near the centre of the channel, over $1 \mathrm{~m} / \mathrm{s}$, and the greater velocity gradient towards the channel wall. At the rear of the gas slug in Figure 6(a) it is evident that the wake of the gas slug is also stronger, given the presence of negative axial velocity values 
(reverse flow) and large velocity gradients. In the case of $\theta=90^{\circ}$, the gas slug and liquid plug wakes are milder and more comparable, resulting in a region where the fluid phases and the interface separating them move at approximately $U_{\text {out }}(0.432 \mathrm{~m} / \mathrm{s})$ up to the proximity of the channel wall. For this case the velocity profiles within the gas slugs and liquid plugs also resemble each other in terms of magnitudes and gradients.

Similar results are also obtained by observing the flow field across the outlet channel cross-sectional plane (y-z) at the $\mathrm{x}$-centroid of the gas slug (Figure 7). In this case the relative axial velocity $\left(v_{x, r e l}\right)$ is plotted, where $v_{\mathrm{x}, \text { rel }}=v_{\mathrm{x}}-U_{\text {out }}$. Complementary to this figure is the graph shown in Figure 8, which provides the axial velocity values (both $v_{\mathrm{x}}$ and $v_{\mathrm{x}, \mathrm{rel}}$ ) across the diagonal channel plane (the same as in Figure 6) also at the $\mathrm{x}$ centroid of the gas slug. Here the greater velocity gradient for the case of $0^{\circ}$ contact angle is also seen, marked by the larger areas corresponding to the extremities of the velocity range at the slug centre and near the channel wall in Figure 7(a), and the steeper curve shape in Figure 8. These figures also allow insight into the liquid flow at the channel corners. It can be seen in Figure 8 that the axial velocity for the $\theta=0^{\circ}$ case along the first 20 micrometers from the corners is nearly zero. This suggests a stagnant liquid, whereas the gas phase only approaches stationary velocity near the gas-liquid interface. As a result of this, it is natural for the average gas phase velocity to exceed the average liquid phase velocity, causing the velocity slip phenomenon reported in Santos and Kawaji (2010). For the case of $\theta=90^{\circ}$, however, there is no possibility for velocity slip to occur since the gas phase completely fills the channel cross-section; as such gas slugs and liquid plugs must move at the same average speed.

These flow profiles suggest internal gas slug recirculation is enhanced in hydrophilic channels. This effect appears to be due to the narrower area the gas slug must travel through when stagnant liquid is present in the channel corners and/or as a liquid film. Given that gas velocity also must approach the low liquid velocity at the interface, to conform to no-slip condition, the gas velocity at the channel center must be high to maintain the overall flow rate, thus producing a larger velocity gradient and greater recirculation compared to the hydrophobic case. Enhanced fluid mixing can improve heat and mass transfer from the channels walls, countering the reduced contact area with channel walls described earlier as a result of slug shape. These results can aid in the design of microfluidic devices, given mixing in microchannels is always a point of concern. 


\section{Conclusions:}

The present study aimed at investigating the effect of the contact angle on slug formation in a microchannel T-junction during gas-liquid two-phase flow by three-dimensional numerical simulation. Microfluidic multiphase computational fluid dynamics is a young field, and therefore it is of interest to highlight innovative approaches used in the present work. One was the introduction of techniques for tracking gas slugs and liquid plugs to obtain precise flow characterization including volume and average slug velocity, whereas available literature often limits analysis to lengths and flow fields.

In the present analysis, it was found that the value of the contact angle has a noticeable effect on the gas slug formation studied numerically. It was observed that as the contact angle increased the gas/liquid interface changed from a convex to a concave shape. The contact angle also affected the velocity profiles of both the gas and liquid phases. By studying the cross-sectional velocity profiles, which is only possible in numerical simulation, it was possible to gain new insight into the fluid dynamics of two-phase microfluidic flow. For a completely hydrophilic channel, the velocity gradients within the gas slug were found to be greater, inducing more internal mixing. At the same time, hydrophilic walls induce the retention of near-stagnant liquid at the

channel corners. This stationary liquid contributes to the occurrence of velocity slip (i.e. higher gas slug velocity and lower void fraction). In the case of partially or fully hydrophobic channels, the gas slug occupies the entire channel cross-section, and therefore no velocity slip occurs. Moreover, in this case the velocity gradients within gas and liquid plugs are attenuated, especially in the wake of the gas slug.

The results reported here agree reasonably well with flow pattern and hydrodynamic observations of Ide et al. (2008) and Serizawa et al. (2002). The results suggest that the use of hydrophobic channels is ideal for increasing the contact area of gas slugs with channel walls, whereas hydrophilic channels are ideal for increasing internal mixing within gas slugs and liquid plugs. Further work is warranted on investigating which of these two contrasting effects improves the wall-slug heat transfer characteristics the most. Nevertheless, the findings in the present work are useful for the design of microfluidic devices, and increase the relevance and usefulness of numerical simulation in the microfluidic field. 
Furthermore, future numerical modeling could implement an advanced model for tracking the moving contact line at the wall to ensure there are no issues with the prediction of the interface dynamics. Rosengarten et al. (2006) suggested that because the contact line must move along the wall, which uses a noslip boundary condition for fluid flow, the VOF code cannot be truly grid independent. The FLUENT code, which uses the model of Brackbill et al. (1992), allows for the movement of the contact line by computing the mass fluxes from integration over the whole cell adjacent to the wall. More modern approaches such as that

developed by Renardy et al. (2001) for VOF CFD code, or that more recently implemented by Chen et al. (2009) for bubble growth and detachment from an orifice, can provide a more accurate means of inducing numerical slip through the introduction of dynamic contact angle treatment and slip boundary condition. Refinement of the numerical mesh near the wall boundaries, which was not considered in the present study, is also ideal for close study of the liquid film thickness, and especially the effect of increasing contact angle on the drainage of liquid film leading to the dry-out phenomena. While such increase in computational cell numbers should increase computational expense dramatically, ever-increasing processor speeds and parallel computing solutions should afford opportunities for such investigation.

\section{Acknowledgements}

R.M Santos is grateful for the financial support received from the Natural Sciences and Engineering Research Council of Canada (NSERC).

\section{References:}

Barajas, A.M., Panton, R.L. (1993). The Effects of Contact Angle on Two-Phase Flow in Capillary Tubes, Int. J. Multiphase Flow, 19, 337-346.

Brackbill, J.U., Kothe, D.B., Zemach, C. (1992). A Continuum Method for Modeling Surface Tension, J. Comp. Phys., 100, 335-354.

Chen, Y., Mertz, R., Kulenovic, R. (2009). Numerical simulation of bubble formation on orifice plates with a moving contact line, Int. J. Multiphase Flow, 35, 66-77. 
Chung, P.M.-Y., Kawaji, M., Kawahara, A., and Shibata, Y. (2004). Two-phase flow through square and circular microchannels - Effects of channel geometry, J. Fluids Eng., 126, 546-552.

Dussan, E.B. (1979). On the Spreading of Liquids on Solid Surfaces: Static and Dynamic Contact Lines, Ann. Rev. Fluid Mech., 11, 371-400.

Fang, C., Steinbrenner, J.E., Wang, F-.M., Goodson, K.E. (2010). Impact of wall hydrophobicity on condensation flow and heat transfer in silicon microchannels, J. Micromech. Microeng., 20, 045018.

Feng, X., Yi, Y., Yu, X., Pang, D-.W., Zhang, Z-.L. (2009). Generation of water-ionic liquid droplet pairs in soybean oil on microfluidic chip, Lab Chip, 10, 313-319.

Gupta, R., Fletcher, D.F., Haynes, B.S. (2009). On the CFD modelling of Taylor flow in microchannels, Chem. Eng. Sci., 64, 2941-2950.

Hirt, C.W., Nichols, B.D. (1981). Volume of Fluid (VOF) Method for the Dynamics of Free Boundaries, J. Comput. Phys., 39, 201-225.

Ide, H., Kimura, R., Inoue, K., Kawaji, M. (2008). Effect of Wetting on Adiabatic Gas-Liquid Two-Phase Flow in a Microchannel, Proceedings of the Sixth International ASME Conference on Nanochannels, Microchannels and Minichannels, Darmstadt, Germany.

Irandoust, S., Andersson, B. (1989). Liquid film in Taylor flow through a capillary, Ind. Eng. Chem. Res., 28, $1684-1688$.

Kandlikar, S.G., Lu, Z., Rao, N., Sergi, J., Rath, C., Dade, C., Trabold, T., Owejan, J., Gagliardo, J., Allen, J., Yassar, R.S., Medici, E., Herescu, A. (2010). Visualization of Fuel Cell Water Transport and Performance Characterization under Freezing Conditions, Information Bridge: DOE Scientific and Technical Information, DOE/GO/17018, doi:10.2172/989206.

Kawahara, A., Chung, P.M.-Y., Kawaji, M. (2002). Investigation of two-phase flow pattern, void fraction and pressure drop in a microchannel, Int. J. Multiphase Flow, 28, 1411-1435.

Kumar, V., Vashisth, S., Hoarau, Y., Nigam, K.D.P. (2007). Slug flow in curved microreactors: Hydrodynamic study, Chem. Eng. Sci., 62, 7494-7504. 
Moita, A.S.H., Moreira, A.L.N. (2003). Influence of surface properties on the dynamic behaviour of impacting droplets, $9^{\text {th }}$ International Conference on Liquid Atomization and Spray Systems, Sorrento, Italy.

Ody, C.P. (2010). Capillary contributions to the dynamics of discrete slugs in microchannels, Microfluid. Nanofluid., 9, 397-410.

Özkan, F., Hecht, K., Pfeifer, P., Schubert, K., Kraushaar-Czarnetzki, B. (2010). Influence of the contact angle on two-phase flow in microreactors for nitrobenzene-hydrogen-stainless steel/carbon, Surf. Interface Anal., 42, 1122-1127.

Raj, R., Mathur, N., Buwa, V.V. (2010). Numerical Simulations of Liquid-Liquid Flows in Microchannels, Ind. Eng. Chem. Res., 49, 10606-10614.

Renardy, M., Renardy, Y., and Li, J., (2001). Numerical Simulation of Moving Contact Line Problems Using a Volume-of-Fluid Method, J. Comput. Phys., 171, 243-263.

Rosengarten, G., Harvie, D.J.E., Cooper-White, J. (2006). Contact angle effects on microdroplet deformation using CFD, Appl. Math. Modell., 30, 1033-1042.

Santos, R.M., Kawaji, M. (2010). Numerical modeling and experimental investigation of gas-liquid slug formation in a microchannel T-junction, Int. J. Multiphase Flow, 36, 314-323.

Santos, R.M., Kawaji, M. (2011). Gas-Liquid Slug Formation at a Rectangular Microchannel T-Junction: A CFD Benchmark Case, Cent. Eur. J. Eng., 1, 341-360.

Schäffer, E., Wong, P.-Z. (2000). Contact line dynamics near the pinning threshold: A capillary rise and fall experiment, Phys. Rev. E, 61, 5257-5277.

Serizawa, A., Feng, Z., Kawara, Z. (2002). Two-phase flow in microchannels, Exp. Therm Fluid Sci., 26, 703-714.

Skartsis, L., Khomami, B., Kardos, J.L. (1992). The effect of capillary pressure on the impregnation of fibrous media, SAMPE J., 28, 19-23.

Takei, G., Nonogi, M., Hibara, A., Kitamori, T., Kim, H-.B. (2007). Tuning microchannel wettability and fabrication of multiple-step Laplace valves, Lab Chip, 7, 596-602.

Wenzel, R.N. (1936). Resistance of Solid Surfaces to Wetting by Water, Ind. Eng. Chem., 28, 988-994. 
West, G.D. (1911). On resistance to the motion of a thread of mercury in a glass tube, Proc. R. Soc. A, 86, 2025 .

Yong, Y.M., Yang, C., Jiang, Y., Joshi, A., Shi, Y.C., Yin, X.L. (2011). Numerical simulation of immiscible liquid-liquid flow in microchannels using lattice Boltzmann method, Sci. China Chem., 54, 244-256. Young, T. (1805). An Essay on the Cohesion of Fluids, Philos. Trans. R. Soc. London, 95, 65-87. 


\section{LIST OF FIGURES}

Figure 1: Young's contact angle configuration.

Figure 2: Computational T-junction mesh at $\mathrm{x}-\mathrm{y}$ plane cross-section.

Figure 3: Effect of contact angle on slug formation.

Figure 4: Static pressure profile around fast and slow gas slugs.

Figure 5: Cross-sectional effect of contact angle $(\theta)$ on liquid film formation.

Figure 6: Axial velocity contour across diagonal channel plane (mirrored-symmetry).

Figure 7: Axial relative velocity contour across cross-sectional (y-z) plane (mirrored-symmetry).

Figure 8: Axial velocity across diagonal channel plane at gas slug x-centroid.

\section{LIST OF TABLES}

Table 1: Effect of contact angle on flow parameters. 
Figure 1

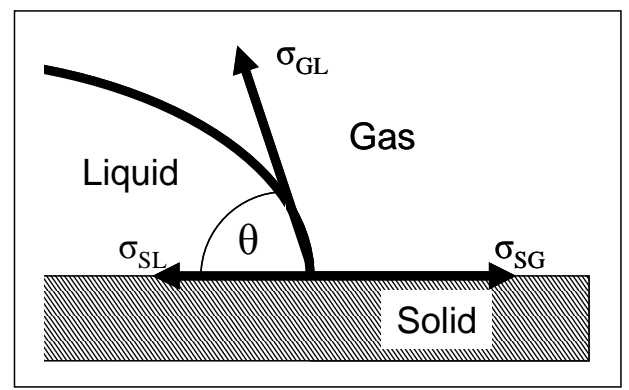


Figure 2

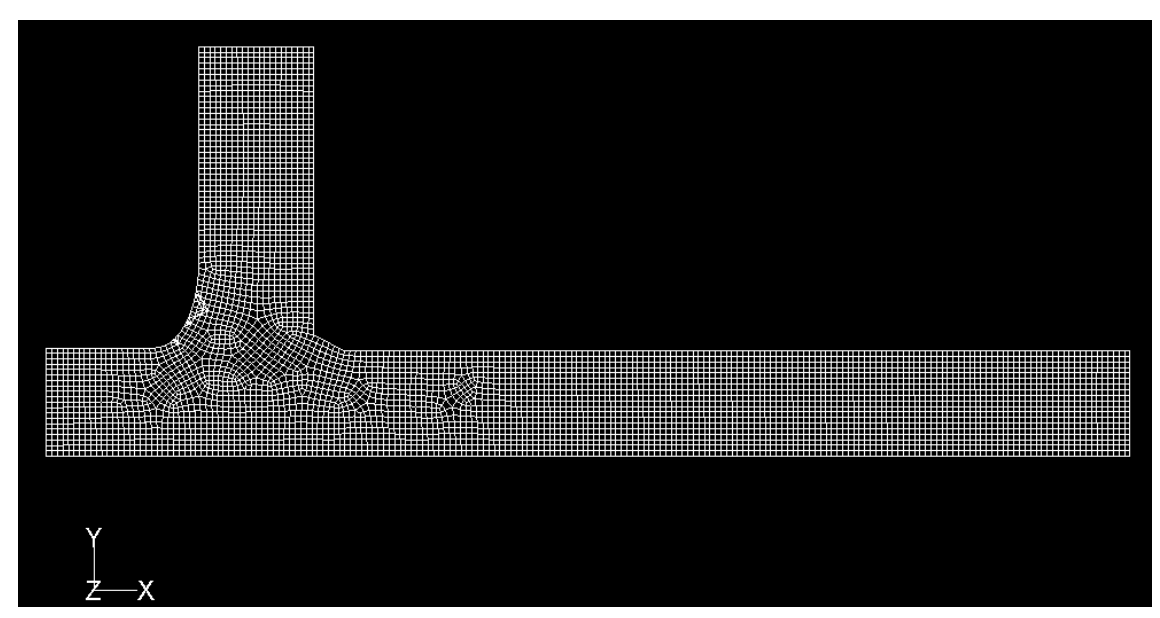


Figure 3

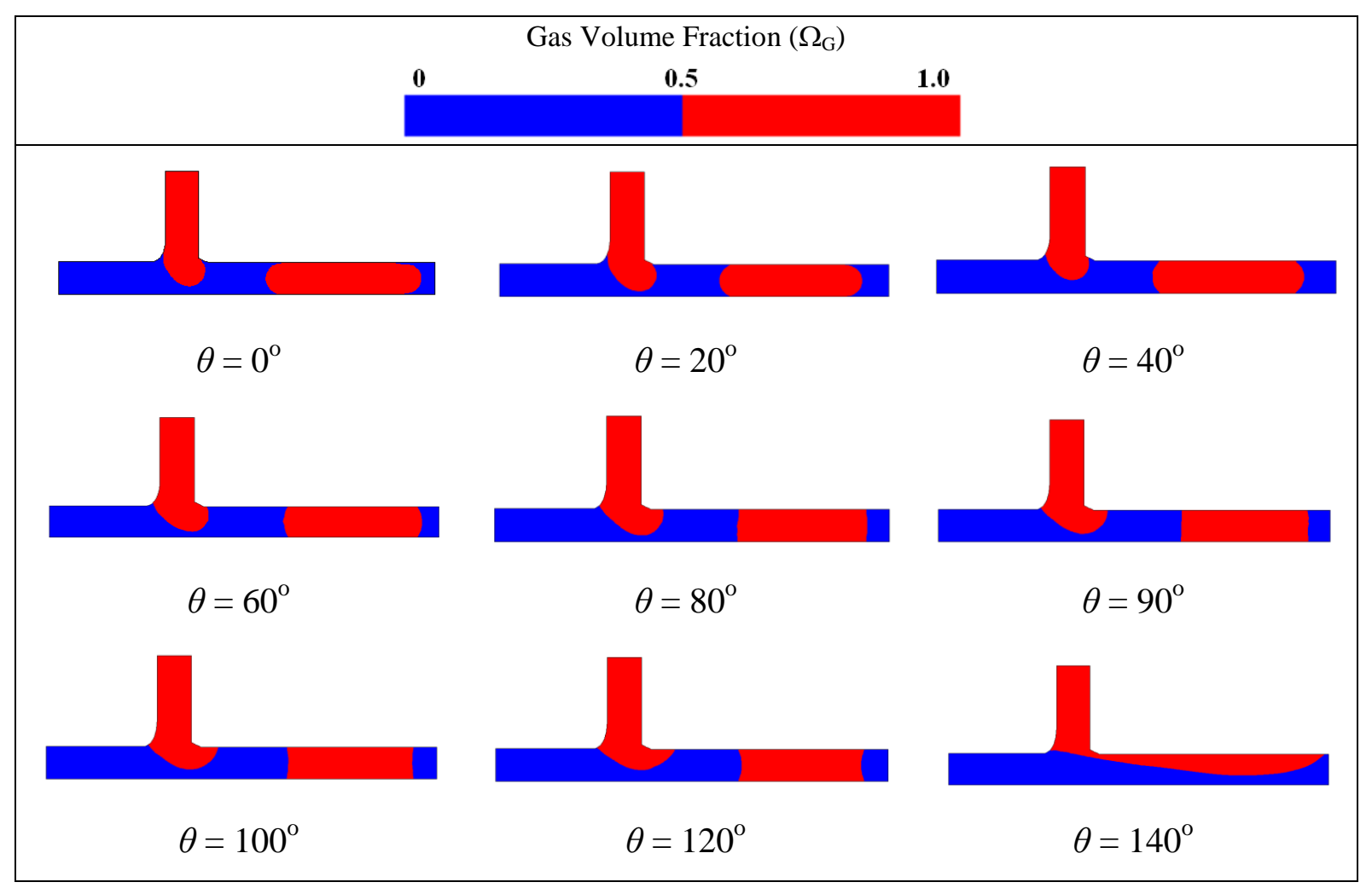




\section{Figure 4}

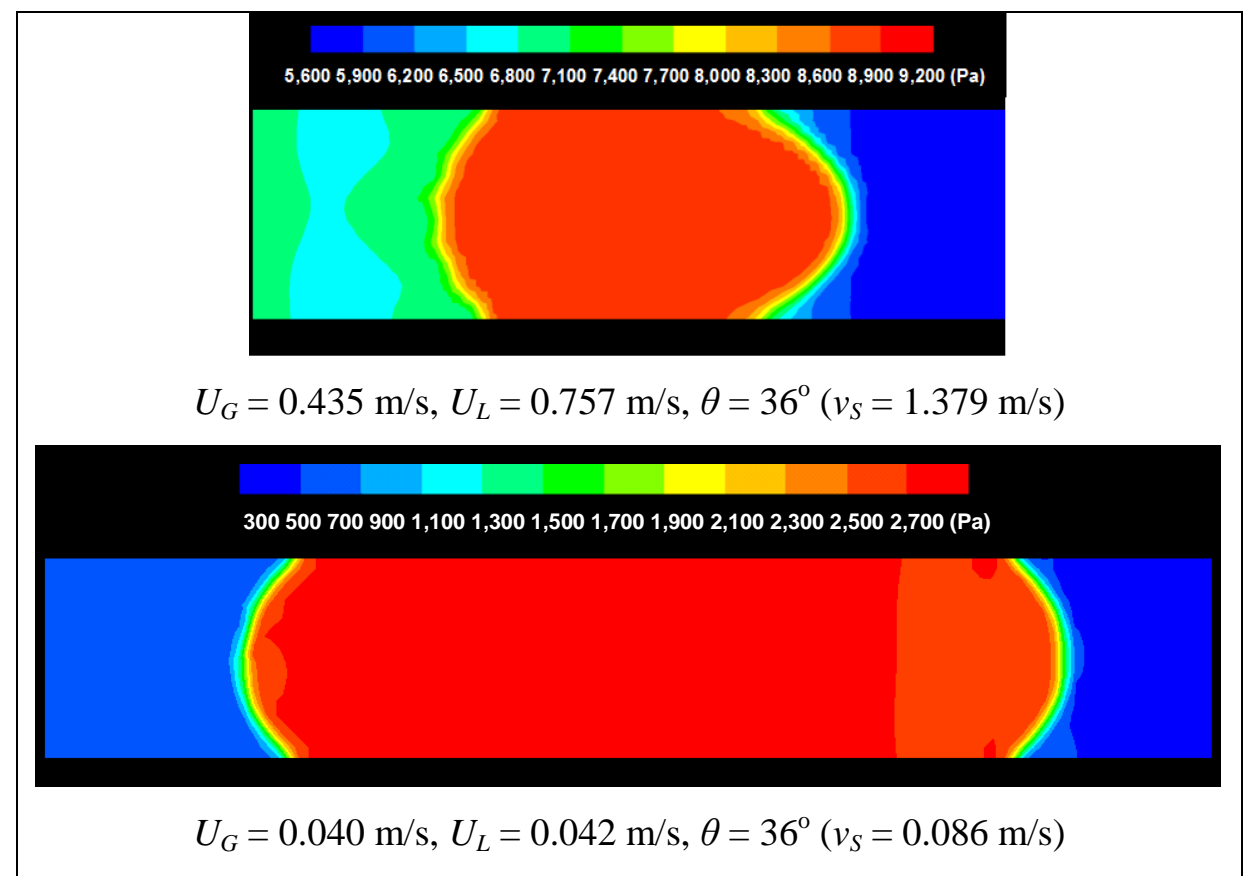


Figure 5

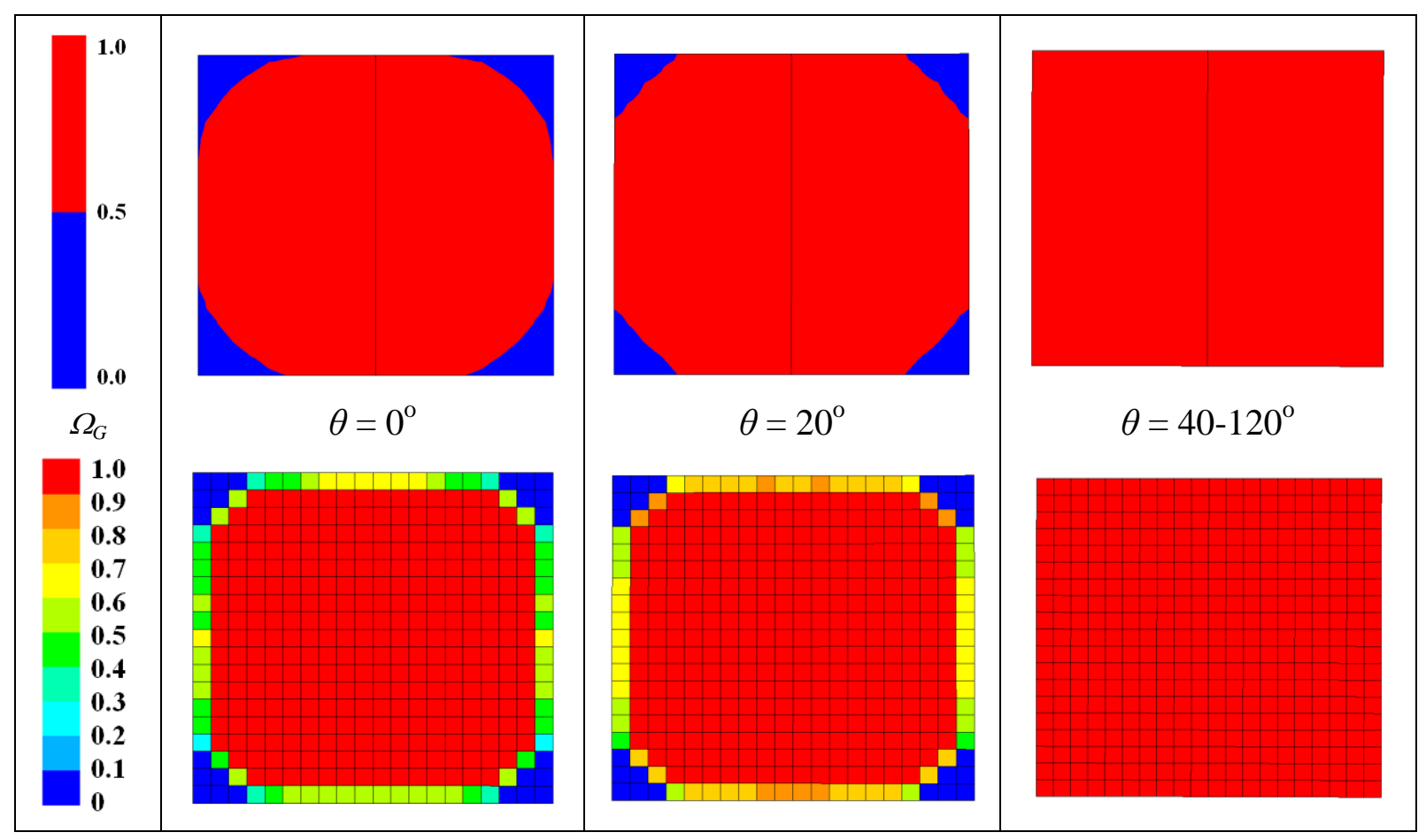


Figure 6

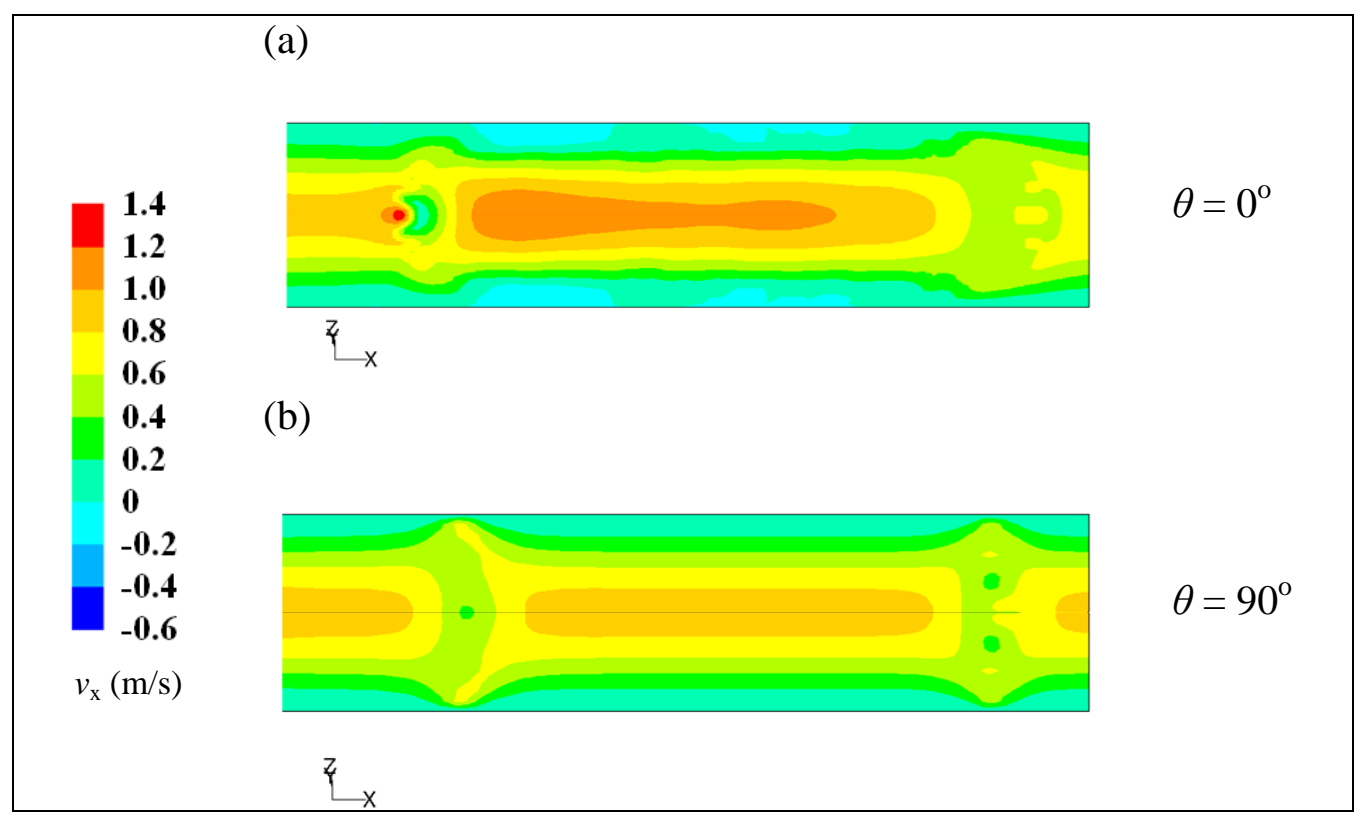


Figure 7

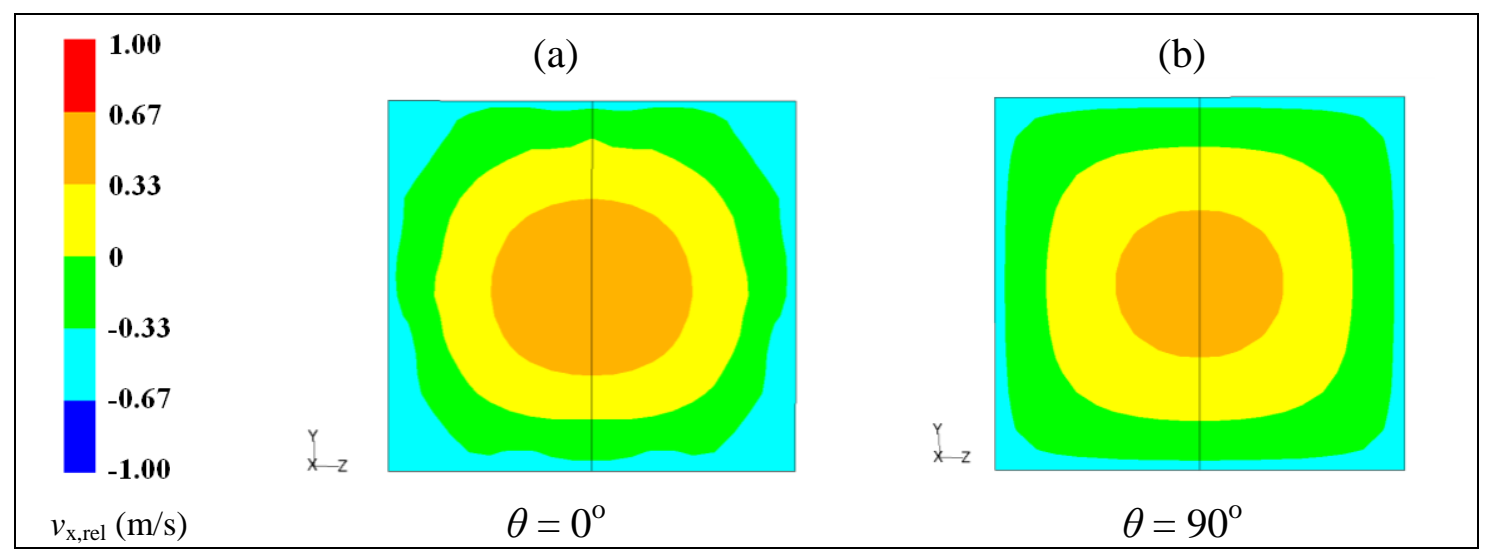




\section{Figure 8}

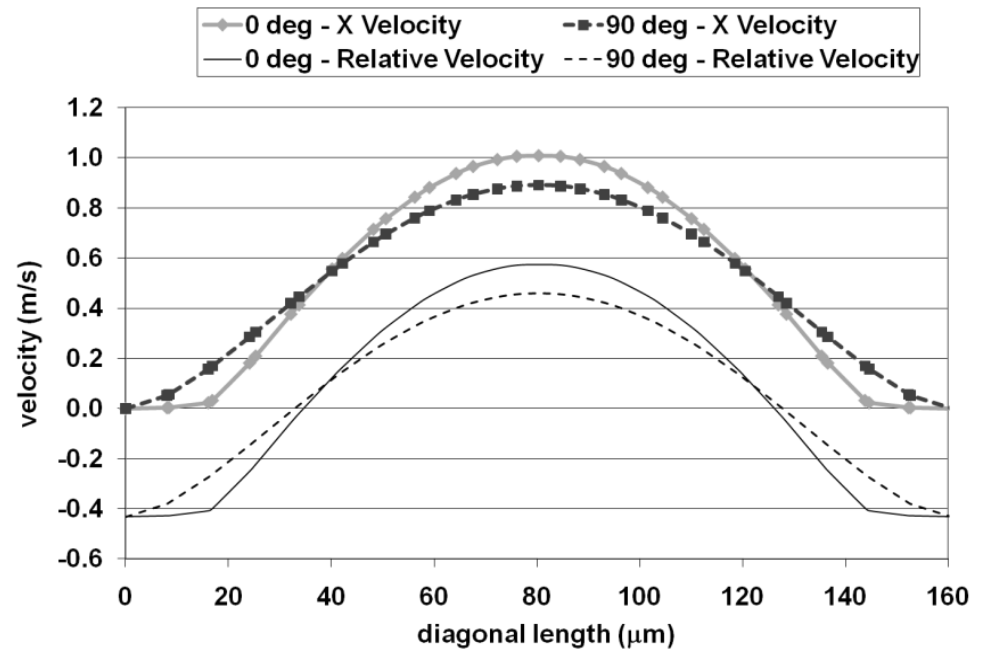


Table 1

\begin{tabular}{cccccc}
\hline$\theta$ & $v_{\mathrm{S}}(\mathrm{m} / \mathrm{s})$ & $V_{S}(\mathrm{~nL})$ & $L_{S}(\mu \mathrm{m})$ & $A_{G}(\%)$ & $t(\mathrm{~s})$ \\
\hline $0^{\circ}$ & 0.506 & 5.50 & 550 & $85 \%$ & 0.0018 \\
$20^{\circ}$ & 0.482 & 5.05 & 487 & $89 \%$ & 0.0018 \\
$40^{\circ}$ & 0.439 & 5.87 & 499 & $100 \%$ & 0.0020 \\
$60^{\circ}$ & 0.432 & 5.80 & 470 & $100 \%$ & 0.0022 \\
$80^{\circ}$ & 0.432 & 5.56 & 437 & $100 \%$ & 0.0022 \\
$90^{\circ}$ & 0.432 & 5.55 & 431 & $100 \%$ & 0.0022 \\
$100^{\circ}$ & 0.432 & 5.52 & 437 & $100 \%$ & 0.0022 \\
$120^{\circ}$ & 0.432 & 5.43 & 448 & $100 \%$ & 0.0022 \\
\hline$U_{\text {out }}$ & 0.432 & & & & \\
\hline
\end{tabular}

